\title{
Auflösung
}

\section{Hämorrhagischer Lichen sclerosus et atrophicus}

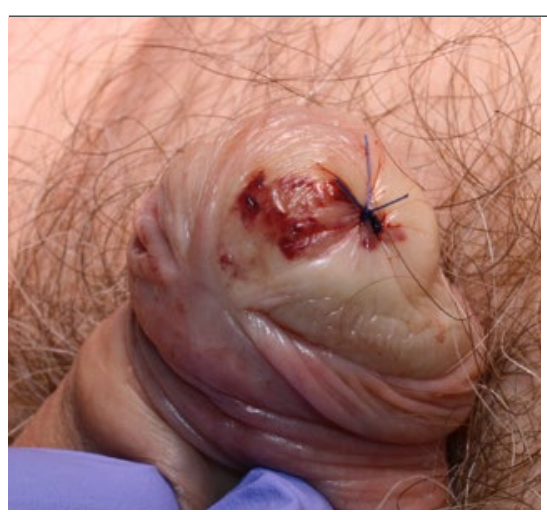

Durch Abhebung der Epidermis entstehen hämorrhagische Blasen.

$\mathrm{D}$ er Lichen sclerosus et atrophicus findet sich bei erwachsenen Männern am häufigsten an der Glans penis und am Präputium, bei Frauen im vulvären Bereich. Die Erscheinungsform am männlichen Genitale wurde erstmals 1908 von Delbanco beschrieben. Die Entwicklung eines spinozellulären Karzinoms ist möglich.

\section{Ätiologie}

Die Ursache des Lichen sclerosus et atrophicus wird kontrovers diskutiert. Eine genetische Komponente spiegelt sich in der familiären Häufung wider. Es wird diskutiert, ob außerdem sowohl autoimmune (gleichzeitiges Auftreten von zum Beispiel Alopecia areata, Vitiligo) als auch infektiöse (Borrelia burgdorferi, HPV) Faktoren eine Rolle spielen.

\section{Diagnose}

Oft handelt es sich um eine Blickdiagnose mit weißlichen Läsionen, bei Abhebung der Epidermis können teils hämorrhagische Blasen (wie im vorliegenden Fall) entstehen. Die Diagnose wird histologisch bestätigt.

\section{Differenzialdiagnose}

Differenzialdiagnostisch muss zum Beispiel an einen Lichen ruber planus, eine Leukoplakie und ein spinozelluläres Karzinom gedacht werden. Beim extragenitalen Lichen sclerosus kommt differentialdiagnostisch eine zirkumskripte Skerlodermie in Frage. Bei Manifestation an der Vulva von Mädchen muss, insbesondere bei Hämorrhagien, der sexuelle Missbrauch als Ursache abgegrenzt werden.

\section{Therapie}

Zur topischen Therapie wird die Anwendung von hochpotenten glukokortikoid- haltigen Salben empfohlen. Die lokale Behandlung mit Calcineurin-Inhibitoren (Tacrolimus) ist möglich.

Bei extragenitalem Lichen sclerosus werden auch Erfolge bei der Bestrahlung mit UVB-Licht einer Wellenlänge von $311 \mathrm{~nm}$ und der Kombination von Psoralenen mit UV-A-Licht (PUVA) beschrieben. Bei ausgeprägten Fällen kann ein Versuch mit Acitretin oral unternommen werden.

Eine operative Therapie kommt vor allem dann in Betracht, wenn der Verdacht auf ein spinozelluläres Karzinom besteht. Bei Herden an der Glans penis ist eine Zirkumzision meist nicht $\mathrm{zu}$ vermeiden, da eine Phimose so verhindert beziehungsweise behandelt und vorhandene Herde zur Abheilung gebracht werden können.
Dr. med. Lea Wiskemann
Dr. med. Benjamin Durani
Dr. med. Hendrike Durani
Dr. med. Nina Scheiba
Dr. med. Sven Bolz
Prof. Wolfgang Hartschuh
Haut- und Laserzentrum Heidelberg
Bergheimer Straße 56a
69115 Heidelberg

\section{Mehr zum Thema auf springermedizin.de}

Eine Übersicht über penile Dermatosen findet sich hier:

- www.springermedizin.de/5858712

Die Differenzialdiagnose von sexuellem Missbrauch bei Kindern ist schwierig.

Eine Übersicht in der Monatsschrift Kinderheilkunde zeigt Beispiele:

- www.springermedizin.de/5583628 